http://jmscr.igmpublication.org/home/

ISSN (e)-2347-176x ISSN (p) 2455-0450

crossref DOI: https://dx.doi.org/10.18535/jmscr/v9i5.35

Journal Of Medical Science And Clinical Research

IGM Publication

An Official Publication of IGM Publication

\title{
Pregnancy Associated Atypical Hemolytic Uremic Syndrome: A Rare Case Report
}

\author{
Authors \\ Dr Manvi Katoch ${ }^{1}$, Dr Tanvi Katoch ${ }^{2 *}$ \\ ${ }^{1}$ Anaesthesiologist, Zonal Hospital, Dharamshala, H.P. \\ ${ }^{2}$ Senior Resident, Department of Obstetrics and Gynecology, PGI, Chandigarh \\ *Corresponding Author \\ Dr Tanvi Katoch
}

Senior Resident, Department of Obstetrics and Gynecology, PGI, Chandigarh, India

\begin{abstract}
Atypical hemolytic uremic syndrome has a genetic predisposition. Pregnancy is a trigger to develop the disease and is a rare entity. It leads to hemolysis in microvasculature, acute renal failure, and thrombocytopenia. The disease is associated with a poor prognosis and needs early treatment initiation. 34-year-old $P_{4} L_{2}$ with post-partum hemorrhage was managed at our centre following which she had acute kidney injury, thrombocytopenia, and repeated fall in hemoglobin. A diagnosis of P-aHUS was made after ruling out other differentials and she was managed with hemodialysis and plasma exchange.

Keywords: pregnancy, atypical hemolytic uremic syndrome, eculizumab, plasma exchange.
\end{abstract}

\section{Introduction}

Atypical hemolytic uremic syndrome (p-aHUS) is a rare disease that is defined by the triad of "microangiopathic hemolyticanemia, thrombocytopenia, and acute kidney injury." $5 \%$ to $10 \%$ cases of hemolytic -uremic syndrome are accounted by aHUS. When thrombotic microangiopthy (TMA) is triggered by pregnancy, the disease is known as pregnancy -associated atypical hemolytic -uremic syndrome (p-aHUS). According to Das et al and Noris et al, "the incidence of aHUS reported is in 1 out of 25,000 pregnancies, after delivery." $[1,2]$

Atypical hemolytic uremic syndrome is difficult to be diagnosed during the puerperial period. Also, it is associated with poor prognosis. Early diagnosis and prompt management initiation are important for improving the outcomes. The treatment for it involves replacement of the mutant type proteins formed by complement pathway, through plasma exchange. ${ }^{[3]}$ Because of its complicated pathogenesis, being a vicious cycle, it is mostly associated with poor maternal outcomes.

\section{Case Report}

A 34-year-old $\mathrm{P}_{4+2}$ woman presented to us with bleeding per vaginumon day-0 post-partum following a full term normal vaginal delivery of male child. On admission, she had normal blood pressure and had no abnormal neurological symptoms. Her antenatal period was uneventful, and she had a normal course of pregnancy without any complications. Uterotonics were used to manage atonic post-partum hemorrhage. There 
was no traumatic component on local vaginal exploration and vaginal packing was done. She had mild pallor, pulse-120 beats/minute, BP104/74mmHg. Her hemoglobin was $6.9 \mathrm{~g} / \mathrm{dL}$, TLC was 20,600 cells/microL, platelet count was $99,000 / \mathrm{mm}^{3}$, renal functions were normal, liver functions were: total bilirubin $2.1 \mathrm{mg} / \mathrm{dL}$, SGOT 104, SGPT 99. Probable diagnosis of hemorrhagic shock vs HELLP vs puerperal sepsis was kept by Obstetrics team. She was managed with crystalloids \& blood transfused. Investigations were sent. At night, patient developed pulmonary edema \& was not able to maintain oxygen saturation.

Patient was taken to Intensive Care Unit. She was then intubated and put-on ventilator with SIMV mode. She was managed with broad spectrum antibiotics, diuretics, central venous pressure guided fluid therapy. On postpartum day-2, patient developed severe oliguria, severe thrombocytopenia, anemia, elevated liver enzymes and acute kidney injury suggesting multi-organ dysfunction. However, there was no fever.

Laboratory investigations revealed $\mathrm{Hb}-8.9 \mathrm{~g} / \mathrm{dL}$, WBC-11.37 cells/microL, platelet count$48,000 / \mathrm{mm}^{3}, \mathrm{BUN}-35 \mathrm{mg} / \mathrm{dl}$, serum creatinine-2.6 mg/dl, LDH-1200U/L, T.bilirubin- 8.4 mg/dl, S. bilirubin-6.10 mg/dl, SGOT- 224 U/L, SGPT-188 U/L， aPTT-35sec,PT-16.6,INR-1.32， D-dimer $>2.0 ;<4.0$. Peripheral smear revealed marked schistocytosis. On examination-pallorand icterus were present, along with oliguria, though uterus was retracted and PPH had resolved. Nephrology consultation was taken, and possibility of ischemic insult $\&$ acute kidney injury was made. Despite all care, patient's condition did not improve in first $48 \mathrm{hrs}$. On postpartum day-3, patient developed anuria and serum creatinine peaked at $3.4 \mathrm{mg} / \mathrm{dL}$. Thrombocytopenia persisted (platelet count $39,000 / \mathrm{mm}^{3}$ ). Liver function tests were improving gradually.

Patient was a booked and well supervised antenatal case with no significant prenatal history. After getting detailed history, we searched for other differential diagnosis for hepatic and renal derangements. Patient received 6 cycles of hemodialysis throughout her stay in hospital. Hemoglobin levels were maintained above $8 \mathrm{~g} / \mathrm{dL}$ with transfusion of PRBCs and 3 cycles of plasma exchange were also given. Patient was afebrile throughout her treatment. She was weaned off the ventilator on day 5. Her blood culture, urine culture was sterile throughout and viral markers (IgM hepatitis A,B,C,E) and scrub typhus reports were negative. On postpartum day 15 , despite 6 cycles of hemodialysis and 3 cycles of plasma exchange, serum creatinine was $4 \mathrm{mg} / \mathrm{dL}$ and platelet count was still low $\left(45,000 / \mathrm{mm}^{3}\right)$. Patient's attendants refused for any further medical management and left against medical advice.

A diagnosis of aHUS was considered due to atypical presentation of "microangiopathic hemolyticanemia,"“thrombocytopenia" and "acute kidney injury" in the postpartum period. ADAMTS13 levels could not be assessed due to unaffordability of the patient.

\section{Discussion}

$\mathrm{P}$-aHUS is a grave systemic disease which is associated with unchecked alternative complement pathway activation.

The disease causes diffuse endothelial injury with formation of fibrin and platelet microthrombi in the micro-vasculature resulting in thrombocytopenia, hemolysis and end organ dysfunction. Its pathogenesis involves uncontrollably activated alternate complement pathway, causing diffuse endothelial damage, thereby resulting in platelet activation, and finally thrombotic angiopathy (TMA) which leads to multi-organ dysfunction and failure.

The diagnosis of $\mathrm{p}$-aHUS is difficult to make, as it resembles many other diseases and thus, they should be ruled out when making a diagnosis. Common features such as acute renal failure, thrombocytopenia, and microangiopathic hemolyticanemia which occur in aHUS are also seen in severe pre-eclampsia with HELLP syndrome, thrombotic thrombocytopenic purpura 
(TTP), and acute fatty liver of pregnancy. HELLP syndrome usually resolves after delivery and our patient's blood pressure was normal. So, it was ruled out in our case study. Patient had no neurological symptoms, no purpura, no fever, thus diagnosis of thrombotic thrombocytopenic purpura (TTP) was also ruled out. Acute fatty liver of pregnancy usually occurs in $3^{\text {rd }}$ trimester of pregnancy with complaints of nausea and vomiting. It generally resolves in 1-2 days of delivery, whilst aHUS usually sets in post-partum period.

Due to destructive nature of aHUS, it is recommended to startplasma exchange within $24 \mathrm{hrs}$ of diagnosis without waiting for genetic testing (eg,ADAMTS13), as genetic testing usually takes weeks. Plasma exchange should be done as soon as possible and should be continued till laboratory parameters normalise well (platelets $>1,50,000 / \mathrm{mm}^{3}$ for 2 consecutive days and $\mathrm{LDH}$ levels fall to normal value).

Eculizumab has been seen to effecting in the treatment of aHUS. It is a humanized recombinant monoclonal antibody and works by inhibiting terminal pathway of complement activation by blocking activation of complement protein C5. Recent studies suggest, "it increases platelet counts, improves renal function, decreases the need for renal replacement therapy, and improves overall quality of life." ${ }^{[4]}$ However, most of the patients are unable to procure its benefit, due to its extremely high price.

\section{Conclusion}

Pregnancy associated atypical hemolytic uremic syndrome is rare. The diagnosis of aHUS is difficult as it often resembles other pregnancy related diseases.

Early diagnosis and early initiation of management are important.
Mainstay of treatment is plasma exchange as early as possible and to involve multi-disciplinary team including nephrologists, hematologists, transfusion medicine experts and obstetricians. Eculizumab should be given if patient can afford.

\section{References}

1. Dashe JS, Ramin SM, Cunningham FG. The long term consequences of thrombotic microangiopathy in pregnancy. Obstet Gynecol 1998;91(5):662-668

2. Noris M, Remuzzi G. Hemolytic uremic syndrome. J Am Soc Nephrol 2005; 16(4):1035-1050

3. Noris M, Remuzzi G. Atypical hemolytic uremic syndrome. N Engl J Med 2009; 361(17):1676-1687

4. Legendre CM, Licht C, Muus $\mathrm{P}$, et al. Terminal complement inhibitor eculizumab in atypical hemolytic-uremic syndrome. N Engl J Med 2013;368 (23):2169-2181. 